\title{
Obesity and associated lifestyle behaviours in Iran: findings from the First National Non-communicable Disease Risk Factor Surveillance Survey
}

\author{
Roya Kelishadi ${ }^{1, *}$, Siamak Alikhani ${ }^{2}$, Alireza Delavari ${ }^{3}$, Farshid Alaedini ${ }^{4}$, \\ Afshin Safaie ${ }^{5}$ and Eliyeh Hojatzadeh ${ }^{6}$ \\ 'Isfahan Cardiovascular Research Center, WHO - Collaborating Center in the EMR, Isfahan University of \\ Medical Sciences, PO Box 81465-1 148, Isfahan, Iran: ${ }^{2}$ Non-communicable Disease Risk Factor Surveillance \\ Office, Center for Disease Control, Ministry of Health and Medical Education, Iran: ${ }^{3}$ Endocrinology \& \\ Metabolism Research Center, Tehran University of Medical Sciences, Iran: ${ }^{4}$ Health Researchers Research and \\ Development Institute, Iran: ${ }^{5}$ Office for Laboratory Affairs, Center for Disease Control, Ministry of Health and \\ Medical Education, Iran: ${ }^{6}$ Cardiovascular Health Office, Center for Disease Control, Ministry of Health and \\ Medical Education, Iran
}

Submitted 17 October 2006: Accepted 29 March 2007: First published online 12 July 2007

\begin{abstract}
Objective: To assess the national prevalence of overweight and obesity, as well as some associated lifestyle behaviours, for the first time in Iran.

Design and Settings: This population-based study was performed in early 2005 as part of the World Health Organization (WHO) STEPwise approach to noncommunicable diseases' risk factor surveillance. Dietary and physical activity habits were assessed by WHO questionnaires.

Subjects: The study population comprised 89532 subjects aged over 15 years living in the 28 provinces of Iran.

Results: Overall, $50.4 \%(n=45113)$ of the participants were male and $64.6 \%$ ( $n=57866$ ) were from the urban areas. The national estimates of overweight, obesity and morbid obesity were $28.6 \%, 10.8 \%$ and $3.4 \%$, respectively. Body mass index $(\mathrm{BMI}) \geq 25 \mathrm{~kg} \mathrm{~m}^{-2}$ in men, women, urban residents and rural residents were found in 37\%, 48\%, 46.7\% and 35.5\%, respectively. Abdominal obesity was present in $43.4 \%$ of women, $9.7 \%$ of men, $28.5 \%$ of the urban residents and $23 \%$ of the rural residents. Overweight as well as generalised and abdominal obesity were more prevalent in the 45-64-year age group. Although there was no significant difference in frequency of consumption of the food groups in subjects with different BMI categories, various kinds of physical activities showed a steady decline with increasing BMI.

Conclusions: The findings of the present study provide alarming evidence for health professionals and policy makers about the very high prevalence of generalised and abdominal obesity in Iran. The unhealthy lifestyle habits, notably sedentary lifestyles in our community, are the major contributing factors for this emerging public health problem.
\end{abstract}

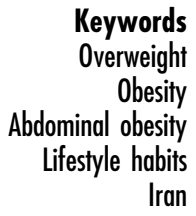

Keywords

Obesity

lifinal obesity

Iran
According to the World Health Organization (WHO) estimates, chronic diseases will account for approximately three-quarters of all the deaths in the developing world by the year $2020^{1}$.

In this regard, the increasing incidence of overweight and obesity could be an emerging public health problem in the low- and middle-income countries. As a result, new cases of the metabolic syndrome may appear, which is likely to create enormous socio-economic and public health burdens for poorer nations in the near future. In countries undergoing epidemiological transition, a complex picture relating to increased food consumption and concomitant changes towards sedentary lifestyles is frequently found ${ }^{2}$. The effects of these changes are particularly severe in the non-European populations with a 'thrifty genotype' and those with an inherited metabolic predisposition to adverse body fat-patterning, i.e. abdominal obesity ${ }^{3,4}$.

Similar to many other developing countries, Iran has been experiencing a rapid phase of urbanisation and industrialisation in recent decades 5 . Limited studies have been performed in certain cities in Iran and they have 
shown differing prevalences of obesity. However, for a vast country like Iran with a great diversity in sociodemographic and lifestyle factors in different provinces, these studies are too small and nationally unrepresentative.

Consequently, we performed the present large population-based survey at national level as the baseline survey of the first surveillance system for the risk factors of non-communicable diseases (NCDs) in Iran, and to the best of our knowledge, in the Eastern Mediterranean region (EMR). This paper presents our findings about the national prevalence of overweight, generalised and abdominal obesity, as well as some associated lifestyle behaviours such as dietary and physical activity habits.

\section{Methods}

This population-based study was performed in early 2005 among 89532 subjects who were over 15 years old and lived in the 28 provinces of Iran. The methodology has been published before ${ }^{6}$. A summary will be presented here.

According to the WHO recommendation, a basic NCD risk factor surveillance site should include a minimum of 2500 participants across the age range of 15-64 years (equal to 250 participants in each 10-year age group). The sample size of the Iranian NCD risk factor surveillance system has been estimated to fulfil this recommendation. Thus, the minimum sample size was taken for provinces with a population of less than 250000 . A larger sample size was calculated for more populated provinces, with a maximum sample size of about 10000 individuals in Tehran province. Participants were selected by multistage random cluster sampling. Each cluster was a block in this study.

A software was developed to randomly identify the starting point to approach households of the blocks through Iran's national databank of ten-digit postal zip codes. Data were collected from 20 habitants in each cluster, including two males and two females in each age group, through interview by trained interviewers using a quota approach.

The instrument of the study was a questionnaire that was mainly adopted from the core and the expanded questionnaires of WHO STEPwise approach to NCD risk factor surveillance ${ }^{7}$. Several optional questions on dietary behaviours were adopted from some national studies to investigate the consumption of dairy products, fried foods and fast foods ${ }^{8}$.

Two questions investigated the frequency of consuming fruits and vegetables in a regular week, followed by questions about the number of servings per day. Also, three questions were added to find out the frequency of consuming dairy products, fried foods and fast foods per week. In addition, 11 questions were adopted from the core questionnaire of WHO STEPwise approach to NCD risk factor surveillance to study the physical activities of the subjects at their jobs, in travelling and during leisure activities. These questions investigated the frequency, intensity and duration of physical activities. Thus, the frequency of vigorous- and moderate-intensity physical activities, which last for at least 10 min continuously, was investigated.

Ethics committee and other relevant national regulatory organisations approved the study. After the full explanation of the procedure involved, written informed consents were obtained from the participants.

Strict training processes and vigorous quality assurance programmes were used to ensure the quality of data collection. The selected individuals were invited to the survey centres for clinical examinations and to answer questions about their sociodemographic and healthrelated characteristics. The questionnaires were filled out confidentially under the supervision of trained nurses. A team consisting of expert health care professionals carried out the field examinations by standardised and calibrated instruments. Height was measured with participants standing without shoes to the nearest $0.5 \mathrm{~cm}$ using a secured metal ruler, while weight was measured in light clothing using calibrated scales. Body mass index (BMI) was calculated as weight divided by height squared $\left(\mathrm{kg} \mathrm{m}^{-2}\right)$. Waist circumference (WC) was measured at a level midway between the lower rib margin and the iliac crest to the nearest $0.5 \mathrm{~cm}$.

Underweight, normal weight, overweight, obesity and morbid obesity were defined as $\mathrm{BMI}<18,18 \leq \mathrm{BMI} \leq$ $24.9,25 \leq \mathrm{BMI} \leq 29.9,30 \leq \mathrm{BMI} \leq 39.9$ and $\mathrm{BMI} \geq 40 \mathrm{~kg} \mathrm{~m}^{-2}$, respectively ${ }^{1}$. The cut-off point for abnormal WC was $\geq 102 \mathrm{~cm}$ for men and $\geq 88 \mathrm{~cm}$ for women?

The intensity of physical activities was categorised by using the following definitions:

1. Vigorous-intensity physical activity: activities that require hard physical effort and cause large increase in breathing or heart rate, e.g. carrying or lifting heavy loads, digging or construction work, running, strenuous sports and weight lifting.

2. Moderate-intensity physical activity: activities that require moderate physical effort and cause small increase in breathing or heart rate, e.g. brisk walking, carrying light loads, light bicycling and fast walking.

3. Work site activity: physical activity at the times the individual spent doing work, such as paid or unpaid work, household chores, harvesting food, fishing, or hunting for food and seeking employment.

4. Transportation activity: physical activity when travelling to or from places, such as going to work or shopping.

5. Leisure-time activity: physical activity in leisure time, i.e. recreation, fitness and sports.

\section{Statistical analysis}

Data obtained in different provinces were collected in the Center for Disease Control in the National Ministry of 
Health. Descriptive statistics were calculated for BMI and the results are expressed as means. The frequencies are provided as per cent. Data are presented for men and women separately, and in 10-year age groups. To account for sampling effect, prevalence rates for overweight and obesity are estimated using the weighted data. According to the sampling method and the equal sample size in each age group, national estimations are undertaken through proportioning the data using a single reference population estimate for the year 2005. Considering the vast area under study, we divided the provinces to those located in the Northern, Central and Southern parts of the country, because of the similarities in the geographic and economic characteristics in these regions. Then we compared the prevalence of weight disorders in the aforementioned three parts of the country. Data were analysed by SPSS software version 11.5 (SPSS Inc.).

\section{Results}

The study population included 89532 individuals; $50.4 \%$ $(n=45113)$ were male and 49.6\% $(n=44344)$ were female. Also, $64.6 \%(n=57866)$ of the participants were from urban areas. The population was equally distributed in different age groups. Overall, 29.3\%, 54.4\% and 16.3\% of the participants were from the Northern, the Central and the Southern parts of the country, respectively.

Table 1 presents the mean values of the anthropometric measures of the participants according to gender and living area. In all the parts of Iran, BMI and WC values were higher in women and in the urban population. In both genders, the highest and the lowest means of BMI and WC were seen in the populations of the Northern and the Southern parts, respectively. The same picture was observed when the prevalence rates of generalised obesity and abdominal obesity were compared among the three different parts of the country. The prevalences of different BMI categories and of abdominal obesity are depicted in Fig. 1.

The national estimate of overweight and obesity altogether was $42.8 \%$, i.e. overweight, obesity and morbid obesity were seen in $28.6 \%, 10.8 \%$ and $3.4 \%$ of the population, respectively. $\mathrm{BMI} \geq 25 \mathrm{~kg} \mathrm{~m}^{-2}$ in men, women, urban residents and rural residents was found in 37\%, 48\%, 46.7\% and 35.5\%, respectively. Abdominal obesity was present in $43.4 \%$ of the women and $9.7 \%$ of the men. It was also present in $28.5 \%$ of the residents in the urban areas and 23\% of the residents in the rural areas. As presented in Table 2, the prevalence of overweight, obesity and morbid obesity increased with age until the age of 54 years and it declined thereafter, whereas abdominal obesity had a constant rise until the age of 64 years. Overweight, generalised and abdominal obesity were more prevalent in the 45-64-year age group.

Table 1 Mean values of anthropometric measures by gender and living area

\begin{tabular}{lrrrrrr}
\hline & Total & North & Centre & South & Urban & Rural \\
\hline Men & & & & & & \\
Weight (kg) & 69.7 & 70.5 & 70.0 & 67.1 & 71.4 & 66.5 \\
Height (cm) & 170.4 & 170.1 & 170.7 & 169.7 & 171.0 & 169.2 \\
BMl (kg m${ }^{-2}$ ) & 24.0 & 24.4 & 24.0 & 23.4 & 24.4 & 23.2 \\
WC (cm) & 84.3 & 85.1 & 84.4 & 82.7 & 85.6 & 81.9 \\
& & & & & & \\
Women & & & & & & \\
Weight (kg) & 63.0 & 64.2 & 63.0 & 60.7 & 64.3 & 60.6 \\
Height (cm) & 157.4 & 156.9 & 157.7 & 157.2 & 158.0 & 156.4 \\
BMl (kg m${ }^{-2}$ ) & 25.5 & 26.1 & 25.4 & 24.6 & 25.8 & 24.8 \\
WC (cm) & 86.0 & 87.3 & 85.7 & 84.9 & 86.7 & 84.8 \\
\hline
\end{tabular}

$\mathrm{BMI}$ - body mass index; WC - waist circumference.

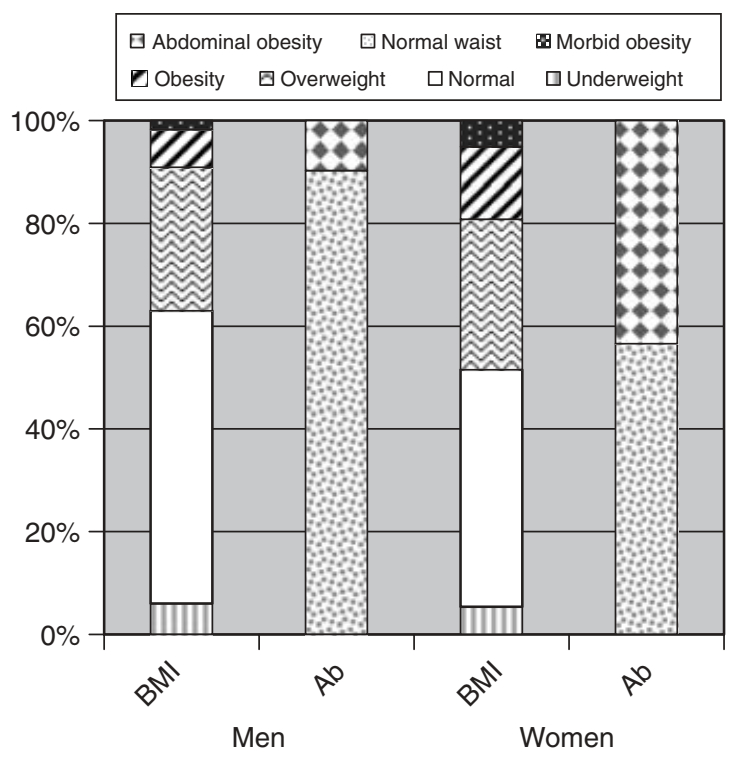

Fig. 1 Prevalence of different categories of body mass index (BMI) and of abdominal obesity (Ab) by gender

Table 2 Prevalence (\%) of generalised and abdominal obesity by age group

\begin{tabular}{lcccccc}
\hline Age groups (years) & Underweight & Normal & Overweight & Obesity & Morbid obesity & Abdominal obesity \\
\hline $15-24$ & 10.8 & 67.0 & 17.1 & 4.0 & 1.2 & 4.0 \\
$25-34$ & 3.4 & 50.4 & 32.3 & 10.8 & 3.1 & 10.8 \\
$35-44$ & 2.1 & 38.6 & 37.1 & 16.7 & 5.4 & 16.7 \\
$45-54$ & 2.1 & 35.3 & 37.8 & 18.5 & 5.4 & 18.5 \\
$55-64$ & 2.5 & 38.6 & 36.6 & 16.9 & 3.4 & 10.8 \\
National estimate & 5.7 & 51.5 & 28.6 & 10.8 & & \\
\hline
\end{tabular}




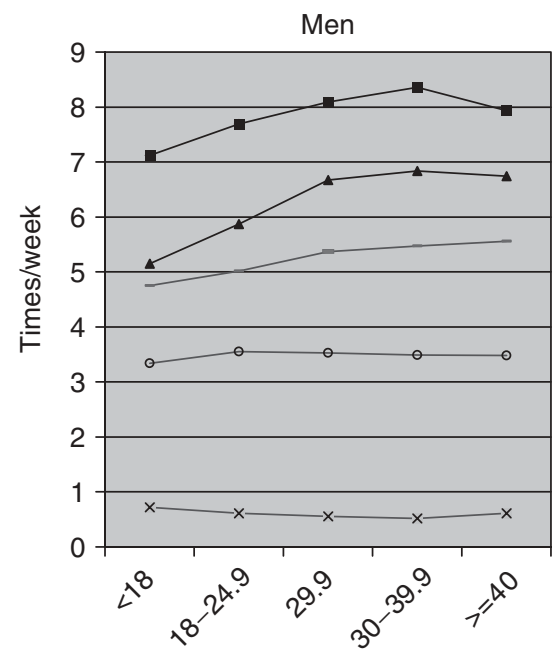

Body mass index $\left(\mathrm{kg} / \mathrm{m}^{2}\right)$

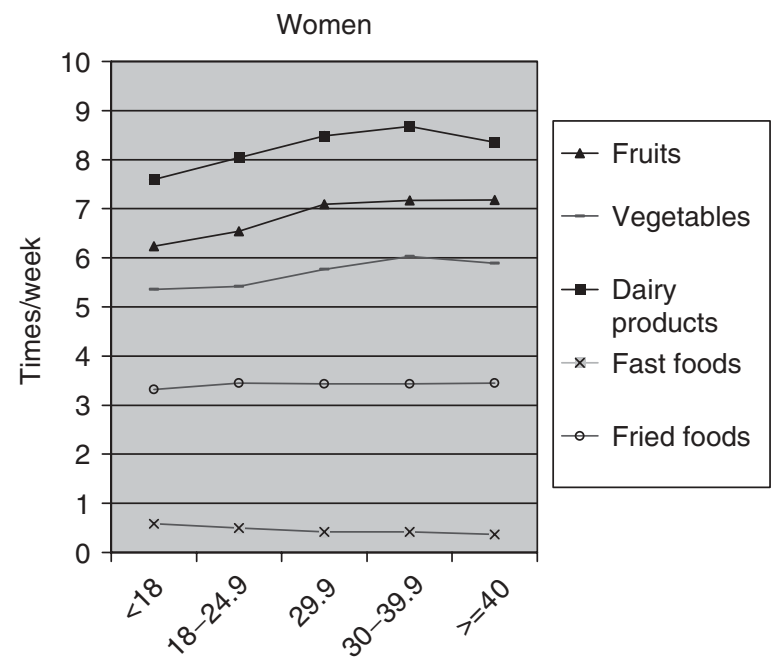

Body mass index $\left(\mathrm{kg} / \mathrm{m}^{2}\right)$

Fig. 2 Frequency (times/week) of consumption of some foods in men and women with different categories of BMI (BMI body mass index)

Although there was no significant difference in the frequency of consumption of food groups among people with different BMI categories (Fig. 2), various kinds of physical activities showed a steady decline with increasing BMI (Fig. 3).

\section{Discussion}

In this large national survey, the first of its kind in Iran and (to the best of our knowledge) in the EMR, we found alarming prevalence rates of generalised and abdominal obesity in our community. Although in recent years concerns have been raised about the problem of obesity in Iran, our findings suggest that our population can expect to see a very high rate of chronic diseases, in the near future notably cardiovascular diseases and diabetes,
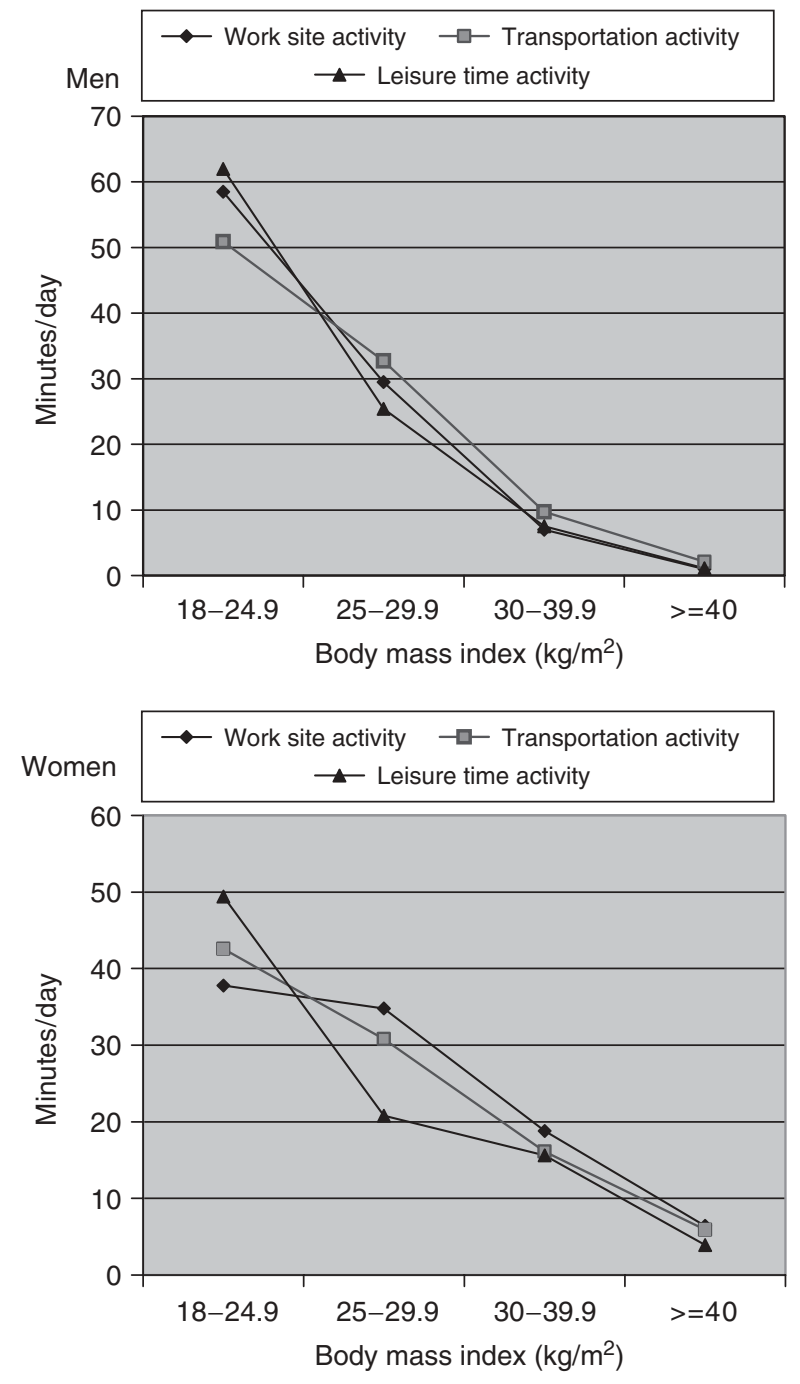

Fig. 3 Daily time (min) spent on physical activity in men and women with different categories of BMI (BMI - body mass index)

as a consequence of the high prevalence of overweight and particularly of abdominal obesity with its recognised serious health outcomes. Neighbouring countries may be experiencing similar increase in their overweight population and they may still grapple with the public health effects of malnutrition and micronutrient deficiencies.

As the Middle East has the highest dietary energy surplus among the developing countries, and considering the rapid changes in the demographic characteristics of the region, large shifts in dietary and physical activity patterns and a rapid rise in risk factors of chronic diseases, especially obesity, are seen accordingly ${ }^{10}$. The International Obesity Task Force reported that the Middle East is one of the regions that has the highest prevalence rates of obesity worldwide ${ }^{11}$. In a study performed in Bahrain, the prevalence of overweight in women and men was reported to be $32 \%$ and $25 \%$, respectively ${ }^{12}$. Moreover, age-specific prevalence of obesity in Bahrainians is higher than that in Saudi adults ${ }^{13-15}$. However, 
rates in Bahrain are similar to the prevalence rates of obesity in Kuwait and in the United Arab Emirates ${ }^{16-18}$. In Oman, the crude prevalence of overweight and obesity $\left(\mathrm{BMI}>25 \mathrm{~kg} \mathrm{~m}^{-2}\right.$ ) reported was $46.2 \%$ for men and $49.5 \%$ for women ${ }^{19}$. In Jordan, the overall prevalence of obesity (BMI $>30 \mathrm{~kg} \mathrm{~m}^{-2}$ ) was reported as high as $49.7 \%$, with a higher prevalence in women than in men, i.e. $59.8 \%$ and $32.7 \%$, respectively ${ }^{20,21}$. A recent national study in Lebanon demonstrated that the prevalence of overweight was higher in men than in women, i.e. $57.7 \%$ vs. $49.4 \%$, respectively, but obesity was more prevalent in women $(18.8 \%)$ than in men $(14.3 \%)^{22}$.

The results of the few studies conducted in some cities of Iran showed large differences. A recent review indicated that the prevalence of overweight (BMI $\geq 85$ th reference percentiles) among the urban 15-39 and 40-69year-olds was $22 \%$ and $40 \%$, respectively. Corresponding values in rural areas were $16 \%$ and $26 \%$, respectively ${ }^{23}$. On the other hand, a study in Tehran showed much higher prevalence rates of generalised and abdominal obesity; $67 \%$ of women and $29 \%$ of the men were obese, and abdominal obesity was detected in $93 \%$ of the women and $74.1 \%$ of the men ${ }^{24}$.

Consistent with some previous studies in the region, in the current population-based study we found higher rates of overweight and obesity, notably abdominal obesity, in women than in men. However, in our study, the prevalence of both generalised and abdominal obesity among men was much lower than found in other studies conducted in some cities in Iran. Based on the extrapolation of our findings, it can be estimated that among the approximately 67 million population of Iran, nearly 29 million are currently overweight and obese. This number illustrates the necessity of an enormous effort to educate the general population and health professionals to identify and properly control this large number of cases. Given the large existing differences in the prevalence of underweight and overweight in different parts of Iran, appropriate practise of evidence-based health promotion requires consideration of both overweight and nutritional deficiencies.

Consistent with some other studies, trends in BMI showed higher estimates up to middle age and a decline thereafter. The lower estimates in older age groups are likely because of weight loss occurring in old age, or alternatively, because of higher risk of mortality for the obese $^{22,25}$. However, in our study, while BMI and generalised obesity increased until 54 years of age, WC and abdominal obesity continued to rise until the age of 64 years.

Our findings about the higher prevalence of obesity in the urban than in the rural residents are in line with some previous studies conducted in the $\mathrm{EMR}^{19,26,27}$. The higher prevalence of overweight and obesity in urban areas confirms the effect of lifestyle on obesity. Availability and low cost of fast foods and unhealthy snacks, sedentary entertainment and less physical activities can explain the higher prevalence of obesity in the urban settings. This is a critical situation because, currently, there is an increasing rate of urbanisation in developing countries, which may have an impact on obesity and its sequels in the future. Some studies have shown the association of physical inactivity and overweight ${ }^{22,28-31}$. Physical activity is a complex behaviour that consists of various components, including leisure time, transportation, hours of work and household activities. Our findings also highlighted the very low levels of physical activity in the population, with much lower levels of all the three types of activities among obese than non-obese individuals. Previous national surveys have shown that nearly $90 \%$ of Iranian women are not physically active ${ }^{32}$. Physical inactivity could well be a major contributor as to why women are more prone to obesity problems than men.

\section{Study limitations and strengths}

The findings of this study should be considered in light of the following limitations. The most important one is probably the recall bias for the process of recording the food intake and the physical activity habits, which requires attention and involves perception; however, the magnitude of this factor is estimated to be low, not acting systematically and possibly exerting a dilution bias. Considering the large number of subjects studied, only a quantitative food-frequency questionnaire was used in the present survey, and such data cannot provide the precise nutrient and energy intake of the participants. Finally, the findings of this study should be interpreted with caution given the cross-sectional nature of the associations.

Despite these factors, this population-based study provides the first national data addressing overweight, generalised and abdominal obesity and the lifestyle behaviours associated with obesity in the Iranian adult population. We used standardised WHO questionnaires and international guidelines for the definition of overweight and classes of obesity, which would allow comparisons with similar reports from other countries. Additional strengths of the study included the strict training of the project team and the vigorous quality assurance programmes that ensured the quality of data collection.

\section{Conclusion}

The findings of the present study provide alarming evidence for health professionals and policy makers about the very high prevalence of generalised and abdominal obesity in Iran. The unhealthy lifestyle habits, notably, the sedentary lifestyles in our community, are major threats for the present and future health of our population and they will make our community prone to an epidemic of chronic diseases in the next two decades. Clearly there is 
a need for a national strategy to tackle the contributors to the excess weight gain at population level.

These findings highlight the necessity of: (1) paying special attention to the elevated future chronic disease risks; (2) establishing a surveillance system for monitoring the time trends in the risk factors of chronic diseases, particularly the trends of obesity based on uniform definitions; and (3) designing programmes to prevent and to control the associated factors in an action-oriented manner.

\section{Acknowledgements}

Sources of funding: This study was conducted by the national Center for Disease Control in the Ministry of Health and the universities of Medical Sciences in different provinces. The study was funded by the Iranian Ministry of Health.

\section{Conflict of interest declaration: None.}

Authorship responsibilities: All authors have participated in the conception of the study as well as in the analysis and interpretation of data.

\section{References}

1 World Health Organization (WHO). Obesity: Preventing and Managing the Global Epidemic, 1st ed. Report of a WHO Consultation on Obesity, Geneva, 3-5 June 1997. WHO/NUT/NCD/98.1. Geneva: WHO, 1998.

2 Hawkes C. Uneven dietary development: linking the policies and processes of globalization with the nutrition transition, obesity and diet-related chronic diseases. Globalization and Health 2006; 2: 4-22.

3 Prentice AM. Early influences on human energy regulation: thrifty genotypes and thrifty phenotypes. Physiology \& Behavior 2005; 86: 640-5.

4 Yusuf S, Reddy S, Ounpuu S, Anand S. Global burden of cardiovascular diseases. Part II. Variations in cardiovascular disease by specific ethnic groups and geographic regions and prevention strategies. Circulation 2001; 104: 2855-64.

5 Ghassemi H, Harrison G, Mohammad K. An accelerated nutrition transition in Iran. Public Health and Nutrition 2002; 5: 149-55.

6 Delavari AR, Alikhani S, Alaedini F. A National Profile of Non-communicable Disease Risk Factors in the I.R. of Iran. Tehran: Center for Disease Control, Ministry of Health and Medical Education, 2005.

7 STEPS Planning and Implementation. STEPS Instruments for NCD Risk Factors (Core and Expanded Version 1.4). Geneva: World Health Organization, 2003.

8 National Nutrition and Health Study, 1999. Tehran: Office for Nutritional Improvement, Ministry of Health and Medical Education, 2000.

9 National Institutes of Health (NIH). Third Report of the National Cholesterol Education Program Expert Panel on Detection, Evaluation, and Treatment of High Blood Cholesterol in Adults (Adult Treatment Panel III), NIH Publication 01-3670. Bethesda, MD: NIH, 2001.

10 Galal O. Nutrition-related health patterns in the Middle East. Asia Pacific Journal of Clinical Nutrition 2003; 12: 337-43.

11 James PT, Leach R, Kalamara E, Shayeghi M. The worldwide obesity epidemic. Obesity Research 2001; 9(Suppl. 4): 228S-33S.
12 Al-Mahroos F, Al-Roomi Kh. Obesity among adult Bahraini population. Annals of Saudi Medicine 2001; 21: 183-7.

13 Al Mannai A, Dickerson JW, Morgan JB, Khalfan H. Obesity in Bahraini adults. Journal of the Royal Society of Health 1996; 116: 30-7.

14 Al-Isa AN. Prevalence of obesity among adult Kuwaitis: a cross-sectional study. International Journal of Obesity and Related Metabolic Disorders 1995; 19: 431-3.

15 Al Nuaim AR, Bamgboye EA, Al Rubeaan KA, Al Mazrou Y. Overweight and obesity in Saudi Arabian adult population: role of sociodemographic variables. Journal of Community Health 1997; 22: 211-23.

16 Al-Isa AN. Changes in body mass index and prevalence of obesity among Kuwaitis 1980-1994. International Journal of Obesity and Related Metabolic Disorders 1997; 21: 1093-9.

17 El-Mugamer IT, Ali Zayat AS, Hossain MM, Pugh RN. Diabetes, obesity and hypertension in urban and rural people of Bedouin origin in the United Arab Emirates. Journal of Tropical Medicine and Hygiene 1995; 98: 407-15.

18 Al-Isa AN. Are Kuwaitis getting fatter? Nutrition and Health 2003; 17: 185-97.

19 Al-Riyami AA, Afifi MM. Prevalence and correlates of obesity and central obesity among Omani adults. Saudi Medical Journal 2003; 24: 641-6.

20 Ajlouni K, Jaddou H, Batieha A. Obesity in Jordan. International Journal of Obesity and Related Metabolic Disorders 1998; 22: 624-8.

21 Centers for Disease Control and Prevention (CDC). Prevalence of selected risk factors for chronic disease-Jordan, 2002. MMWR Morbidity and Mortality Weekly Report 2003; 52: $1042-4$.

22 Sibai AM, Hwalla N, Adra N, Rahal B. Prevalence and covariates of obesity in Lebanon: findings from the first epidemiological study. Obesity Research 2003; 11: 1353-61.

23 Rashidi A, Mohammadpour-Ahranjani B, Vafa MR, Karandish M. Prevalence of obesity in Iran. Obesity Reviews 2005; 6: 191-2.

24 Azadbakht L, Mirmiran P, Shiva N, Azizi F. General obesity and central adiposity in a representative sample of Tehranian adults: prevalence and determinants. International Journal for Vitamin and Nutrition Research 2005; 75: 297-304.

25 Williamson DF. Descriptive epidemiology of body weight and weight change in US adults. Annals of Internal Medicine 1993; 119: 646-9.

26 Abdul-Rahim HF, Holmboe-Ottesen G, Stene LC, Husseini A, Giacaman R, Jervell J, et al. Obesity in a rural and an urban Palestinian West Bank population. International Journal of Obesity and Related Metabolic Disorders 2003; 27: 140-6.

27 Nanan DJ. The obesity pandemic implications for Pakistan. Journal of the Pakistan Medical Association 2002; 52: 342-6.

28 Slattery ML, Sweeney C, Edwards S, Herrick J, Murtaugh M, Baumgartner $\mathrm{K}$, et al. Physical activity patterns and obesity in Hispanic and non-Hispanic white women. Medicine and Science in Sports and Exercise 2006; 38: 33-41.

29 Sakuta H, Suzuki T. Physical activity and selected cardiovascular risk factors in middle-aged male personnel of self-defense forces. Industrial Health 2006; 44: 184-9.

30 Ismail MN, Chee SS, Nawawi H, Yusoff K, Lim TO, James WP. Obesity in Malaysia. Obesity Reviews 2002; 3: 203-8.

31 Firdaus M, Mathew MK, Wright J. Health promotion in older adults: the role of lifestyle in the metabolic syndrome. Geriatrics 2006; 61: 18-22.

32 Sheikholeslam R, Mohamad A, Mohammad K, Vaseghi S. Non communicable disease risk factors in Iran. Asia Pacific Journal of Clinical Nutrition 2004; 13(Suppl.): S100. 